\title{
Cobertura universal em saúde e o Programa Mais Médicos no Brasil
}

\author{
Elisandréa Sguario Kemper ${ }^{1}$, Renato Tasca', Erno Harzheim², Julio M. Suárez \\ Jiménez ${ }^{1}$, Jorge Hadad ${ }^{3}$ e Maria Fátima de Sousa ${ }^{4}$
}

Como citar Kemper ES, Tasca R, Harzheim E, Jiménez JMS, Hadad J, Sousa MF. Cobertura universal em saúde e o Programa Mais Médicos no Brasil. Rev Panam Salud Publica. 2018;42:e1. doi: 10.26633/RPSP.2018.1

RESUMO A cobertura universal pode ser entendida como uma meta que engloba diversas medidas que permitem a ampliação do acesso pelos sistemas de saúde. A atenção primária à saúde (APS) deve ser vista como um aspecto essencial desse processo, com papel de re-organização dos serviços com base nas necessidades em saúde. O Programa Mais Médicos no Brasil traz uma série de medidas para fortalecer a APS no país. A partir de uma revisão conceitual de cobertura universal em saúde e de uma análise do Programa Mais Médicos sob a ótica dos resultados obtidos em termos de fortalecimento da APS no Sistema Único de Saúde (SUS), o objetivo do artigo foi discutir a potencial contribuição do Programa Mais Médicos para o avanço do sistema de saúde brasileiro rumo à cobertura universal. Conclui-se que o Programa Mais Médicos é um propulsor para o alcance da cobertura universal no SUS.

Palavras-chave

Cobertura universal em saúde; Sistema Único de Saúde; atenção primária à saúde; Brasil.

A cobertura universal em saúde pode ser entendida como uma meta que engloba diversas medidas estruturais que permitem aos sistemas de saúde ampliar o acesso e implementar serviços capazes de dar respostas às necessidades em saúde da população. Com a cobertura universal, espera-se obter melhorias nas condições de saúde e, como resultado, promover o desenvolvimento humano, reduzir a

Organização Pan-Americana da Saúde (OPAS)/ Organização Mundial da Saúde (OMS), Brasília (DF), Brasil. Correspondência: Elisandréa Sguario (with a g) Kemper, kempere@paho.org

2 Universidade Federal do Rio Grande do Sul, Faculdade de Medicina, Porto Alegre (RS), Brasil.

3 Organização Pan-Americana da Saúde (OPAS), Montevidéu, Uruguai.

4 Universidade de Brasília, Núcleo de Estudos em Saúde Coletiva, Unidade de Saúde da Família, Brasília (DF), Brasil. pobreza e as desigualdades e melhorar a qualidade de vida.

Entre diversos caminhos para atingir a cobertura universal, a literatura mostra que o foco na qualificação da atenção primária à saúde (APS) potencializa os esforços dos países no sentido de fortalecer os sistemas de saúde. Uma APS de qualidade - isto é, que permita acesso oportuno de todas as pessoas aos bens e serviços de saúde, sem distinção e conforme as necessidades em saúde - compreende a coordenação do acesso a cuidados integrais $(1,2)$, o que inclui necessariamente a presença de médicos na equipe multidisciplinar.

O Programa Mais Médicos (PMM), uma política implantada no Brasil em 2013, prevê o fortalecimento da APS no Sistema Único de Saúde (SUS) por meio de um conjunto de ações que vai desde a alocação de médicos nos serviços até a reordenação da formação médica no país. O objetivo do programa é diminuir a carência de médicos nas regiões prioritárias para o SUS, reduzindo as desigualdades regionais na saúde e adequando os processos de educação e formação médica para fortalecer o SUS.

O presente artigo propõe um diálogo entre a cobertura universal em saúde e o PMM, tendo a APS como elo entre essas políticas. A partir de uma revisão conceitual de cobertura universal em saúde e de uma análise do PMM sob a ótica dos avanços e resultados obtidos em termos de fortalecimento da APS no SUS, o objetivo do artigo foi discutir a potencial contribuição do PMM para o avanço do sistema de saúde brasileiro rumo à cobertura universal. 


\section{ASPECTOS CONCEITUAIS DA COBERTURA UNIVERSAL}

A universalidade é um dos princípios fundamentais do SUS, que determina que todos os cidadãos brasileiros, sem qualquer distinção, tenham direito ao acesso à saúde. O SUS representou uma conquista democrática: antes da implementação do sistema, o acesso aos serviços públicos de saúde era restrito às pessoas com vínculo formal de trabalho. Entretanto, apesar dos avanços conquistados com a criação do SUS e de seu embasamento no princípio da universalidade, o sistema enfrentou dificuldades na implementação, especialmente considerando o contexto político e econômico do país no período da sua regulamentação (3).

A busca por medidas que possam superar as barreiras à universalização segue acompanhando o SUS e os sistemas de saúde de outros países em desenvolvimento. Paralelamente a esses esforços, os encontros mundiais de chefes de Estado vêm produzindo acordos e declarações internacionais que se constituem em compromissos políticos assumidos pelos países e servem como referência para nortear as mudanças nos sistemas de saúde. O documento mais simbólico nesse sentido, sempre atual, foi a Declaração de Alma-Ata, de 1978, que reforça que os cuidados primários de saúde são a chave para atingir a meta da saúde para todos, como parte do desenvolvimento e da justiça social (4).

Pode-se considerar que a cobertura universal não tem uma definição única, podendo ser qualificada como um termo polissêmico e até ambíguo, uma vez que pode adquirir diferentes conotações conforme distintos interesses. Mesmo não existindo consenso, os países continuam implementando reformas nos seus sistemas de saúde a partir do próprio entendimento do que seja cobertura universal, com distintos focos; por exemplo, alguns privilegiam o acesso aos serviços pelos pobres, outros enfocam as iniquidades em saúde e outros ainda ressaltam o controle das doenças (5).

No Brasil, onde o direito a um sistema público e universal de saúde está previsto na Constituição Federal, o debate conceitual acerca da cobertura universal em saúde tem sido intenso. Tem-se discutido também a necessidade de um novo marco, ou de uma nova meta para o SUS, considerando que o sistema já está amparado pelo arcabouço legal do direito à saúde e dos princípios do SUS, entre os quais está explícita a universalidade (6).

Entretanto, é relevante ressaltar que, até o presente momento, o SUS não alcançou a universalidade e a integralidade. Assim, é importante manter na pauta da agenda de cobertura universal em saúde aspectos como financiamento (e sua restrição), mecanismos de prestação de serviços, fornecimento de medicamentos e insumos, força de trabalho em saúde, implementação das redes de atenção, sistemas de informação e comunicação, tecnologias em saúde, mecanismos de garantia da qualidade, governança, legislação e mecanismos de regulação, entre outros.

Nesse contexto, a cobertura universal deixa de ser apenas um princípio para se tornar uma meta. Esse movimento pode servir para mobilizar os países a reformularem as estruturas dos sistemas de saúde de forma a garantir que as pessoas tenham acesso efetivo a ações e serviços como demanda, no caso do Brasil, o arcabouço constitucional do direito à saúde.

\section{CAMINHOS PARA A COBERTURA UNIVERSAL}

Na América Latina, os países têm percorrido caminhos distintos para implementar a cobertura universal, seja por meio de sistemas unificados de saúde, como é o caso do Brasil, Cuba e Costa Rica, seja pela criação de subsistemas paralelos de seguros e de prestação de serviços para grupos populacionais específicos, segundo a situação de trabalho, a exemplo da Argentina, Chile, Colômbia, México, Peru, Uruguai e Venezuela. Todos os países citados, com exceção da Venezuela, tornaram o direito à saúde explícito nas constituições (1).

As reformas para implementar a cobertura universal envolvem mudanças importantes nos sistemas de saúde, exigindo a mobilização de recursos adicionais e a reformulação das estruturas financeiras e organizacionais (7). São obstáculos à cobertura universal a fragmentação da organização e da prestação de serviços, a segmentação e a restrição de financiamento, as falhas na regulação do setor privado e público, as falhas na substituição dos gastos privados por gastos públicos, as dificuldades na eliminação dos gastos com desembolso direto e a permanência de modelos de atenção que não contemplam as características epidemiológicas, demográficas e os determinantes sociais da saúde.

Enquanto a cobertura universal tem ganhado o centro do debate sobre sistemas e serviços de saúde, o componente fundamental que é a APS tem sido negligenciado. A APS representa um marco abrangente para discutir a organização dos sistemas e dos serviços de saúde, podendo se constituir como espinha dorsal do sistema, melhorando os resultados, reduzindo custos e promovendo equidade (8) - e, em última análise, a cobertura universal.

\section{ATENÇÃO PRIMÁRIA À SAÚDE COMO CAMINHO PARA A COBERTURA UNIVERSAL}

A Declaração de Alma-Ata qualificou a APS como o paradigma mais apropriado para alcançar a Saúde para Todos no ano 2000 (4). Difundiu para o mundo a concepção de atenção primária integral, de acordo com o qual a APS é parte do sistema integrado de cuidados em saúde e do desenvolvimento econômico e social da sociedade, reduzindo as iniquidades em saúde, promovendo a participação social e contribuindo para a garantia do direito à saúde. Corroborando essa afirmação, a estratégia para acesso e cobertura universal em saúde para a Região das Américas destaca que o fortalecimento da atenção primária é essencial para o desenvolvimento dos sistemas de saúde, a fim de reduzir as desigualdades por meio da cobertura e acesso universais (9).

Na América Latina, as políticas de APS foram marcadas por modelos seletivos e focalizados, que se propunham a implementar pacotes mínimos de serviços de saúde, dirigidos a grupos populacionais marginalizados. No Brasil, a APS seletiva se tornou hegemônica nos anos 1980 (10). Na década de 1990, os modelos de APS na América Latina foram se desenvolvendo em direção à APS integral. A abordagem biopsicossocial estava se expandindo e trouxe consigo um avanço no acesso aos serviços, mas não o suficiente para a universalização e a efetivação do direito à saúde (2).

A partir dos anos 2000, surgiu um movimento de renovação da APS, reconhecendo que ela vinha sendo interpretada e implantada com base em políticas equivocadas em alguns países. Houve um chamado aos países para produzirem mudanças nos sistemas de saúde com o 
objetivo de implementar a APS integral, coordenadora dos cuidados em uma rede integrada de serviços (11). As redes de atenção à saúde são um importante componente das estratégias de renovação da APS. No centro dessas redes, a APS amplia o acesso, coordena e articula a estruturação dos serviços com base nas necessidades identificadas no primeiro nível, tornando os sistemas mais efetivos e eficientes.

Evidências robustas demonstram que países que possuem sistemas de saúde organizados a partir da APS apresentam melhores resultados, com aumento da efetividade. Dessa forma, afirmam o alto poder da APS na redução das desigualdades em saúde, na maior eficiência do cuidado, na coordenação do fluxo dos usuários no sistema, na satisfação dos usuários e na maior utilização de práticas preventivas. Reforçam ainda o papel da APS como importante estratégia para o enfrentamento dos problemas de saúde, prevenindo mortes e doenças (12-16).

\section{O PROGRAMA MAIS MÉDICOS NO BRASIL}

No Brasil, o fortalecimento da APS tem sido um processo gradativo, vinculado à ampliação da Estratégia Saúde da Família (ESF), que é a forma brasileira de organizar a atenção primária. A ESF está baseada nos princípios do SUS e apoiada nos atributos da APS, como acesso, integralidade, coordenação do cuidado, longitudinalidade, orientação familiar e comunitária (17). Em 20 anos, a ESF ampliou a cobertura em saúde de $5 \%$ para $60 \%$ da população, com impactos positivos tanto para a saúde da população quanto para o sistema de saúde (18). Atualmente, o Brasil possui mais de 40000 equipes de Saúde da Família implantadas e cobertura nominal próxima a 130 milhões de pessoas, o que significa pouco mais de $60 \%$ da população brasileira (19). Os outros $40 \%$ da população ainda são atendidos pelas formas anteriores de organização dos serviços, que não utilizam base territorial nem orientação comunitária e familiar. A diferença entre a qualidade e a efetividade desses dois modelos foi amplamente avaliada, comprovando que a ESF é superior (20).

É possível afirmar que a ESF tem sido uma experiência bem-sucedida; porém, precisa radicalizar na busca dos atributos da APS e efetivar o seu papel central nas redes de atenção como coordenadora do cuidado e na reorientação dos serviços entre os níveis de atenção (21).

Entre os desafios importantes para a APS estão a fixação de profissionais qualificados nas equipes (em especial do médico), o grau de efetividade clínica das equipes frente aos problemas de saúde mais frequentes e a prática efetiva da coordenação do cuidado individual com garantia da integralidade (22).

O número de médicos por habitante é baixo no Brasil se comparado a outros países com sistemas universais de saúde. Além disso, a distribuição dos médicos pelo território nacional é desigual em relação às regiões do país (23). A falta de médicos nos serviços contribui para a baixa valorização da APS, fazendo com que os usuários procurem respostas às suas demandas em serviços de pronto-atendimento, os quais são focados no atendimento por queixa-conduta. Além disso, a falta de médicos é uma das principais razões para a adesão a um plano de saúde suplementar.

Frente a esses desafios foi criado o PMM, instituído pela lei $\mathrm{n}^{\circ} 12$ 871, de 22 de outubro de 2013, como resposta do Governo Federal ao apelo explícito de apoio solicitado pelos gestores municipais para enfrentar a carência de médicos com perfil adequado para a APS no SUS, em especial para compor as equipes de Saúde da Família (24). O PMM trouxe um conjunto de medidas estruturadas em três eixos, um que trata do provimento emergencial de médicos para as equipes de APS, outro da formação, com ações envolvendo a reordenação da oferta de cursos de medicina e de residência médica e um terceiro eixo que trata do investimento em infraestrutura nas unidades básicas de saúde (UBS).

$\mathrm{O}$ eixo provimento emergencial chegou a recrutar e alocar mais de 18 mil médicos, em $73 \%$ dos municípios do país, cobrindo inclusive todas as áreas indígenas. Os médicos do PMM são brasileiros, formados no Brasil ou no exterior, e estrangeiros, oriundos de mais de 40 países. Mais de $60 \%$ são cubanos e fazem parte do acordo de cooperação entre os governos do Brasil e de Cuba, com o apoio da Organização Pan-Americana da Saúde (OPAS)(25).

Os médicos do PMM estão alocados em municípios ou regiões de municípios mais vulneráveis, os quais já têm um histórico de priorização nas políticas do SUS, tais como locais com alto percentual de população em extrema pobreza, baixo índice de desenvolvimento humano (IDH), regiões de árido e semiárido, ou onde vivem populações indígenas, quilombolas e ribeirinhas, entre outras. O PMM contribui para reduzir a carência de médicos em regiões prioritárias e vulneráveis, a exemplo das regiões Norte e Nordeste do país, onde a escassez de médicos reflete a grave condição socioeconômica vivida e que foram as regiões que mais receberam médicos do programa (23).

Os estudos sobre o PMM indicam resultados positivos, principalmente no eixo provimento emergencial (18). Em síntese, esses resultados apontam para ampliação do acesso, da equidade, da satisfação dos usuários e da humanização do cuidado. Foram identificadas práticas inovadoras, mudanças nos processos de trabalho e troca de conhecimentos entre os médicos e as equipes de saúde na produção do cuidado, contribuindo para o aperfeiçoamento das práticas e formação em serviço. Destaca-se a prática do cuidado integral e da longitudinalidade.

A qualidade da infraestrutura das UBS é um fator relacionado à satisfação com o trabalho e está associada à rotatividade dos médicos. As UBS em piores condições têm menores chances de participar dos programas de incentivo federal, o que enfraquece a potencialidade de redução de iniquidades no acesso aos recursos em saúde. Dentre os argumentos para a institucionalização do PMM, a inadequada qualidade da infraestrutura das UBS foi apontada como um dos fatores que limitam a fixação dos médicos na APS (26).

O eixo da formação profissional do PMM tem relevância para a sustentabilidade das mudanças geradas pelo programa, uma vez que o provimento emergencial possui caráter limitado e a consolidação da iniciativa depende da fixação de médicos brasileiros. Esse eixo prevê medidas nos cursos de graduação em medicina, com expansão do número de profissionais formados e alternativas que modifiquem o perfil de formação e incentivem a interiorização dos médicos. Também atua junto aos programas de residência médica propondo ampliação das vagas em medicina de família e comunidade e obrigatoriedade de 1 ano de medicina de família e comunidade para outras especialidades médica (clínica médica, pediatria, ginecologia e obstetrícia, cirurgia geral, psiquiatria e medicina preventiva e social) (27). 


\section{PROGRAMA MAIS MÉDICOS E A COBERTURA UNIVERSAL}

Como considerações finais deste artigo, cabe iniciar pela importância do SUS para o Brasil, representando uma vitória da sociedade brasileira, pela conquista do direito à saúde e pela construção da maior política social do país. Para os países da América Latina, o SUS é uma referência de sistema público universal e um símbolo de resistência, por sobreviver às constantes ameaças impostas pelas muitas barreiras estruturais e conjunturais.

Dentre os desafios que devem ser superados pelo sistema de saúde brasileiro, destacam-se a fragmentação e a segmentação do sistema e dos serviços, a iniquidade no acesso e na cobertura, a carência de recursos humanos com formação adequada às necessidades de mudança do modelo de atenção, a baixa qualidade da atenção e o financiamento insuficiente. Muitos desses desafios comprometem o alcance da cobertura universal em saúde.

Na perspectiva da cobertura universal como meta dos sistemas para concretizarem o direito à saúde, enfatiza-se a APS como melhor caminho para universalizar o acesso com atenção integral e coordenada e para organizar o sistema com base nas necessidades em saúde. Isso porque a APS está próxima das pessoas, inserida nas comunidades e com a maior competência para decodificar e pactuar as necessidades em saúde, sendo mais resolutiva, com maior satisfação dos usuários e respondendo aos aspectos organizacionais do sistema.

O PMM pode ser considerado como uma "mola propulsora" para o desenvolvimento da APS no SUS. Mesmo que o eixo provimento emergencial tenha caráter provisório, deixa o legado de que investir em APS (e, em especial, na ESF, pelo estabelecimento de equipes completas, com médico de família e comunidade) é um dos caminhos para o fortalecimento do sistema de saúde.

É possível afirmar que estruturas adequadas facilitam uma melhor atenção à saúde. A combinação de elementos estruturais e operacionais dos serviços de saúde produz análises que não estão relacionadas com efeitos diretos na saúde da população, mas com os meios para conseguir os melhores resultados em saúde. Dessa forma, considera-se como estruturante o eixo de investimento nas UBS previsto no PMM.

\section{REFERÊNCIAS}

1. Atun R, de Andrade LO, Almeida G, Cotlear D, Dmytraczenko T, Frenz P, et al. Health-system reform and universal health coverage in Latin America. Lancet. 2015;385(9974):1230-47.

2. Anderson MIP, Armadillo MLR, Días NT, Fuentes SC. Cobertura universal en salud, atención primaria y medicina familiar. Rev Bras Med Fam Comunidade. 2016;12(1):4-30.

3. Carvalho G. A saúde pública no Brasil. Estud Av. 2013; 27(78):1-22.

4. Declaração de Alma-Ata. Alma-Ata: Determinantes Sociais da Saúde. 1978. Disponível em: Portal e observatório sobre iniquidades em saúde. http://dssbr.org/ site/documentos-de-referencia/ Acessado em dezembro de 2017.

5. Dmytraczenko T, Almeida G, organizadores. Rumo a uma cobertura universal de saúde e equidade na América Latina e no Caribe. Evidência de países selecionados. Brasília: Banco Internacional para Reconstrução e Desenvolvimento/Banco Mundial; 2016.

6. Centro Brasileiro de Estudos em Saúde. Nota pública sobre cobertura universal em saúde. Disponível em: http://cebes.org. $\mathrm{br} / 2014 / 08 /$ nota-consulta-publica-cobertura-universal-da-saude-da-omsopas-nobrasil Acessado em 14 de fevereiro de 2017.
7. Frenk J. Leading the way towards universal health coverage: a call to action. Lancet. 2015;385(9975):1352-58.

8. Stigler FL, Macinko J, Pettigrew LM, Kumar R, Van Well C. No universal health coverage without primary health care. Lancet. 2016;387(10030):1811.

9. Organización Panamericana de la Salud (OPS). Salud en las Américas, edición del 2017. Washington, D.C.: OPS; 2017. Disponível em: http:/ /www.paho.org/salud-en-las-americas-2017/?page_id=4235. Acessado em 28 de fevereiro de 2017.

10. Giovanella L, Almeida PF, Vega Romero R, Oliveira S, Tejerina Silva H. Panorama de la atención primaria de salud en Suramérica: concepciones, componentes y desafíos. Saúde Debate. 2015;39(105):300-22.

11. Organização Mundial da Saúde (OMS). Relatório Mundial da Saúde 2008. Cuidados primários de saúde: agora mais que nunca. 2008. Disponível em: http:// www.who.int/eportuguese/publications/ pt/ Acessado em 22 de fevereiro de 2017.

12. Macinko J, Guanais FC, de Fátima M, de Souza M. Evaluation of the impact of the Family Health Program on infant mortality in Brazil, 1990-2002. J Epidemiol Community Health. 2006;60(1):13-9.

13. Rasella D, Aquino R, Barreto ML. Impact of the Family Health Program on the
Por sua vez, o eixo formação do PMM carece de mais avaliações para analisar se as estratégias são as mais acertadas para os objetivos a que se propõe. Apesar disso, o PMM tem sido considerado como a intervenção de recursos humanos mais importante na América Latina nos últimos anos (28).

O fortalecimento da APS não pode ser visto somente como um dos caminhos para alcançar a cobertura universal. Deve ser encarado como "o caminho" para ampliar o acesso e efetivar o direito à saúde, tendo em vista as evidências que comprovam que a APS contribui para aumentar a participação e a inclusão dos cidadãos. O PMM parece ser um dos elementos de fortalecimento dessa APS, que pode aproximar o SUS cada vez mais da meta de cobertura universal no Brasil.

Conflitos de interesse. Nada declarado pelos autores.

Declaração. As opiniões expressas no manuscrito são de responsabilidade exclusiva dos autores e não refletem necessariamente a opinião ou política da RPSP / PAJPH ou da Organização Panamericana de Saúde (OPAS). quality of vital information and reduction of child unattended deaths in Brazil: an ecological longitudinal study. BMC Public Health. 2010;10:380.

14. Macinko J, Dourado I, Aquino R, Bonolo Pde F, Lima-Costa MF, Medina MG, et al. Major expansion of primary care in Brazil linked to decline in unnecessary hospitalization. Health Aff (Millwood). 2010;29(12):2149-60.

15. Mendonça CS, Harzheim E, Duncan BB, Nunes LN, Leyh W. Trends in hospitalizations for primary care sensitive conditions following the implementation of Family Health Teams in Belo Horizonte, Brazil. Health Policy Plan. 2012;27(4):348-55.

16. Rasella D, Harhay MO, Pamponet ML, Aquino R, Barreto ML. Impact of primary health care on mortality from heart and cerebrovascular diseases in Brazil: a nationwide analysis of longitudinal data. BMJ. 2014;349:g4014.

17. Starfield B. Is primary care essential? Lancet. 1994;344(8930):1129-33.

18. Kemper ES. Mendonça AVM, Sousa MF. Programa Mais Médicos: panorama da produção científica. Cienc Saude Coletiva. 2016;21(9):2785-96.

19. Brasil, Ministério da Saúde. Histórico de cobertura da Saúde da Família do Portal 
do Departamento de Atenção Básica. Disponível em: http://dab.saude.gov.br/ portaldab/historico_cobertura_sf.php Acessado em 24 de fevereiro de 2017.

20. Van Stralen CJ, Belisário SA, Van Stralen TBS, de Lima AMD, Massote AW, Oliveira CL. Percepção dos usuários e profissionais de saúde sobre atenção básica: comparação entre unidades com e sem saúde da família na Região CentroOeste do Brasil. Cad Saude Publica. 2008;24(1):148-58.

21. Harzheim E, Duncan B, Stein A, Cunha C, Goncalves M, Trindade T, et al. Quality and effectiveness of different approaches to primary care delivery in Brazil. BMC Health Serv Res. 2006;6:156.

22. Organização Pan-Americana da Saúde (OPAS), Ministério da Saúde, Conselho Nacional de Secretários Estaduais de Saúde, Conselho Nacional de Secretários
Municipais de Saúde. Inovando o papel da atenção primária nas redes de atenção à saúde: resultados do laboratório de inovação em quatro capitais brasileiras. Brasília: OPAS; 2011. (NAVEGADORSUS, 3).

23. Oliveira JPA, Sanchez MN, Santos LMP. O Programa Mais Médicos: provimento de médicos em municípios brasileiros prioritários entre 2013 e 2014. Cienc Saude Coletiva. 2016;21(9):2719-27.

24. Brasil, Ministério da Saúde. Lei 12 871/2013. Disponível em: http://www. planalto.gov.br/ccivil_03/_ato20112014/2013/lei/112871.htm Acessado em 10 de dezembro de 2017.

25. Brasil, Ministério da Saúde. Programa Mais Médicos. Disponível em: http:// maismedicos.gov.br Acessado em 28 de fevereiro de 2017.

26. Giovanella L, Mendonça MHM, Fausto MCR, de Almeida PF, Bousquat A, Lima
JG, et al. A provisão emergencial de médicos pelo Programa Mais Médicos e a qualidade da estrutura das unidades básicas de saúde. Cienc Saude Coletiva. 2016;21(9):2697-708.

27. Silva Júnior AG, Andrade HS. Formação médica no Programa Mais Médicos: alguns riscos. Cienc Saude Coletiva. 2016;21(9):2670-1.

28. Harris M. Mais Médicos (More Doctors) Program - a view from England. Cienc Saude Coletiva. 2016;21(9):2919-23.

Manuscrito recebido em 9 de fevereiro de 2017. Aceito em versão revisada em 4 de outubro de 2017.
ABSTRACT

Universal health coverage and the More Doctors physician recruitment program (Programa Mais Médicos) in Brazil

Keywords Universal coverage; Unified Health System; primary health care; Brazil.
Universal health coverage can be understood as a goal encompassing a series of structural measures that allow health care systems to enhance access to health care. Primary health care (PHC) should be seen as an essential component of this process, in charge of re-organizing services according to the health needs of the population. A Brazilian physician recruitment program, Programa Mais Médicos (More Doctors), has introduced a set of measures that strengthen PHC. Based on a conceptual review of universal health coverage and on an analysis of the More Doctor Program from the perspective of the results obtained in terms of strengthening PHC in the public Unified Health System (SUS), the aim of the present article was to discuss the potential contribution of the More Physicians Program to the progress towards universal coverage in the SUS. It is concluded that the More Doctors Programs is a driver of universal coverage in the public health care system in Brazil.
RESUMEN

\section{Cobertura universal de salud y el programa Más Médicos (Programa Máis Médicos) en Brasil}

Palabras clave
La cobertura universal de salud puede entenderse como una meta que abarca una serie de medidas estructurales que permiten a los sistemas de atención médica mejorar el acceso a la atención médica. La atención primaria de salud (APS) debe ser vista como un componente esencial de este proceso, responsable de la reorganización de los servicios, tomando en cuenta las necesidades de salud de la población. El programa Más Médicos, ha introducido en Brasil un conjunto de medidas que fortalecen la APS. Con base en una revisión conceptual de la cobertura universal de salud y de un análisis del programa Más Médicos desde la perspectiva de los resultados obtenidos en términos de fortalecimiento de la APS en el Sistema Único de Salud público (SUS), el objetivo del presente artículo fue analizar la contribución potencial del programa Más Médicos al progreso hacia la cobertura universal en el SUS. Se concluye que el programa Más Médicos es un motor de cobertura universal en el sistema de salud pública en Brasil.

Cobertura universal en salud; Sistema Único de Salud Familiar; atención primaria de salud; Brasil. 